\title{
EL COMPONENTE PRAGMÁTICO EN ADULTOS CON SIINDROME DE ASPERGER: ACTOS DE HABLA INDIRECTOS, METÁFORAS Y COERCIÓN ASPECTUAL
}

\author{
THE PRAGMATIC COMPONENT IN ADULTS WITH ASPERGER \\ SYNDROME: INDIRECT SPEECH ACTS, METAPHORS, AND \\ ASPECTUAL COERCION
}

\author{
CONSTANZA MURRAY \\ Universidad de Chile, Santiago, Chile \\ constanza.murray@gmail.com \\ ANITA TOBAR \\ Universidad de Chile, Santiago, Chile \\ anitatobarh@gmail.com \\ FANNY VILLABLANCA \\ Universidad de Chile, Santiago, Chile \\ fannyvillablancaz@gmail.com

\section{GUILLERMO SOTO} \\ Universidad de Chile, Santiago, Chile \\ gsoto@uchile.cl
}

\section{RESUMEN}

El presente estudio explora el rendimiento de adultos con síndrome de Asperger (SA) en tres tareas lingüísticas con un componente pragmático; a saber, comprensión de actos de habla indirectos, de metáforas y de cláusulas con coerción exocéntrica. Empleando secciones pertinentes de la versión en español del Protocolo para la Evaluación de la Comunicación de Montréal, MEC (Ferreres et al., 2007), y el test de coerción aspectual de Reyes (2012), se comparan el rendimiento de sujetos con $\mathrm{SA}(\mathrm{N}=5)$ y el de neurotípicos $(\mathrm{N}=5)$. Los resultados muestran diferencias en el comportamiento de los adultos con SA y los neurotípicos. Estas parecen obedecer a que, en los primeros, la información contextual no influye positivamente en la comprensión. Se plantea que la diferencia entre ambos grupos podría obedecer a un déficit en la operación del principio de relevancia en los

\footnotetext{
* Este trabajo se enmarca en el proyecto Fondecyt $2011 \mathrm{~N}^{\circ} 1110525$ y se origina en un seminario de grado realizado en 2011 en la U. de Chile (Murray et al., 2012).
} 
adultos con SA. Los resultados son concordantes con tres de las teorías neurocognitivas que caracterizan el SA: la teoría hipersistematizante, la de coherencia central débil y, de modo más indirecto, la de déficit en la teoría de la mente.

Palabras clave: Síndrome de Asperger, pragmática, actos de habla indirectos, metáforas, coerción aspectual.

\begin{abstract}
This study explores the performance of adults with Asperger Syndrome (SA) in three linguistic tasks that involve a pragmatic component: the comprehension of indirect speech acts, metaphors, and clauses with exocentric aspectual coercion. Using the relevant sections of the Spanish version of the Protocole Montréal d'Evaluation de la Communication, MEC (Ferreres et al., 2007), and the aspectual coercion test designed by Reyes (2012), it compares the performance of $\mathrm{SA}(\mathrm{N}=5)$ and neurotypical $(\mathrm{N}=5)$ subjects. The results show that contextual information does not improve the performance of SA subjects. The difference between both groups may result from a deficit in the relevance principle in adults with SA. The results agree with three neurocognitive theories of SA: hypersystemising theory, weak central coherence theory, and, more indirectly, theory of mind deficit.
\end{abstract}

Keywords: Asperger Syndrome, pragmatics, indirect speech acts, metaphors, aspectual coercion.

Recibido: 21.03.2014. Aceptado: 20.03.2015.

\title{
1. INTRODUCCIÓN
}

$\mathrm{Z}^{1}$ Síndrome de Asperger (en adelante, SA) es un trastorno generalizado del Cdesarrollo incluido normalmente entre los trastornos del espectro autista que suele homologarse al autismo de alto funcionamiento ${ }^{1}$. Los trastornos del espectro autista se caracterizan por la presencia de dificultades en la interacción social derivadas de un desarrollo cerebral diferencial (Attwood, 2007). Estas diferencias se dan tanto en el nivel citoarquitectónico, involucrando áreas frontales, témporo-

\footnotetext{
${ }^{1}$ El origen del diagnóstico se remonta a 1944, cuando Hans Asperger introduce el término de 'trastorno autista de la personalidad' para denominar un fenómeno caracterizado por un conjunto de rasgos de desadaptación social, y que, según el autor, formaba parte de un continuum natural de las aptitudes (Attwood, 2007). Al ser descrito como un trastorno del desarrollo que presenta gran capacidad de adaptación y que no involucra necesariamente deterioro cognitivo, no ha habido consenso acerca de si considerarlo o no dentro de los trastornos del espectro autista. Actualmente, se clasifica como parte de ellos por el DSM-V.
} 
parietales y algunas partes del sistema límbico, como en los períodos de desarrollo cerebral, debido a diferencias en la concentración de sustancias bioquímicas en el cerebro (McAlonan et al., 2008; O'Brien et al., 2010; Thompson, Thompson y Reid, 2010) ${ }^{2}$.

Aun cuando no hay consenso en la bibliografía, se ha planteado que, en el plano neurocognitivo, el SA se puede caracterizar por distintos problemas, enunciados en cuatro principales teorías de funcionamiento cognitivo. En primer lugar, la Teoría de la Mente ha asociado el SA a problemas en el desarrollo normal de las habilidades de mentalización, caracterizando la patología principalmente por dificultades para atribuir y empatizar con estados mentales o emocionales de terceros (Baron-Cohen, Leslie y Frith, 1985; Happé, 1994). La Teoría de Coherencia Central, por su parte, ha identificado como un rasgo nuclear del síndrome un funcionamiento anormal de la identificación y manejo del sentido global del discurso (Frith, 2003; Happé, 1999; Norbury, 2005). La Teoría Disejecutiva, en cambio, aboga por la centralidad de problemas en el correcto funcionamiento del sistema ejecutivo, los cuales impedirían realizar normalmente tareas relacionadas con memoria de trabajo, atención y otras funciones ligadas a la corteza prefrontal (Pellicano, 2007). Finalmente, la Teoría Hipersistematizante afirma que el SA se caracteriza principalmente por una tendencia a hipersistematizar la realidad, es decir, a la identificación y generación de reglas que faciliten la comprensión y manejo de información que, para personas sin el diagnóstico, no implican un costo cognitivo adicional (Baron-Cohen, 2002; Baron-Cohen y Belmonte, 2005).

Las personas con SA presentan una serie de problemas que afectan el uso del lenguaje, entre éstos, dificultades en la atribución de intenciones e identificación de los estados mentales de otros, en la comprensión de lenguaje no-literal, y en la comprensión y uso de las pautas de interacción social (Attwood, 2007; Segura, 2007; Valdez, 2005; Loukusa y Moilanen, 2009; Le Sourn-Bissaoui, Caillies, Gierski y Motte, 2009). No obstante, los sujetos con SA, en relación con el resto de los sujetos del espectro autista, poseen una gran capacidad de adaptación ${ }^{3}$, por lo que con una adecuada estimulación pueden alcanzar conductas en gran medida similares a las de un sujeto neurotípico ${ }^{4}$.

${ }^{2}$ Como, por ejemplo, $\mathrm{N}$-acetil aspartato, creatina y colina.

${ }^{3}$ A diferencia de los demás trastornos del espectro autista, el SA no está necesariamente asociado a un déficit cognitivo (Segura, 2007). Si bien es cierto que algunos sujetos presentan problemas cognitivos, hay también casos en que se presenta un nivel de inteligencia mayor que el promedio. La causa de esta amplitud de posibilidades no ha sido determinada, pero podría estar relacionada con las diferencias de estímulo o con los problemas asociados que presente el sujeto (i. e., funciones ejecutivas, coherencia central, teoría de la mente o hipersistematización).

${ }^{4}$ El término 'neurotípico' se acuña en la comunidad autista, a propósito de la comprensión de las diferencias de desarrollo cerebral, como un signo de neurodiversidad más que como la mera evidencia de la presencia de un trastorno o de un problema de desarrollo. Desde este punto de vista, no hay un modelo humano único del cual cada persona es una versión, sino que la especie está formada por todos y cada uno de los individuos que la componen y, por lo tanto, incluye todas las diferencias 
En los últimos años se ha desarrollado una gran cantidad de estudios sobre el lenguaje en personas con SA (Atwood, 2007; Ghaziuddin y Gerstein, 1996; Hadjikhani, 2007; Happé, 1999; Kaland et al., 2002; Shriberg et al., 2001; Le SournBissaoui et al., 2009; Lewis, Murdoch y Woodyatt, 2007; Loukusa y Moilanen, 2009; McAlonan et al., 2008; O’Brien et al., 2010; Pijnacker, Hagoort, Buitelaar, Teunisse y Geurts, 2008; Rivière y Castellanos, 2003; Segura, 2007; Thompson et al., 2010; Valdez, 2005). Estas investigaciones han mostrado que, si bien parecen presentar una morfosintaxis y una semántica normales (pero cfr. Osorio, 2014), tienen importantes trastornos en el componente pragmático y discursivo. En particular, se ha estudiado su comportamiento social y emocional (Attwood, 2007), la capacidad para hacer inferencias pragmáticas (Pijnacker et al., 2008), el desarrollo de la teoría de la mente (Kaland et al., 2002), la prosodia (Shriberg et al., 2001) y lo que se ha denominado "habla pedante" (Ghaziuddin y Gerstein, 1996). Si bien el perfil lingüístico y pragmático de los sujetos con SA no es homogéneo, se han observado dificultades en el nivel suprasegmental, en el uso de verbos mentales, en la comprensión de lenguaje figurado y expresiones idiomáticas, en la variedad de actos de habla empleados y, de modo más general, en aquellos casos en que la comprensión demanda una fuerte integración de la información lingüística y contextual (para un perfil más detallado, v. Osorio, 2014).

No obstante el creciente interés por el estudio del lenguaje en personas con SA, no son muchas las investigaciones centradas en adultos. Esto parece deberse a que el diagnóstico es relativamente reciente, la sintomatología suele atribuirse a otros trastornos y, finalmente, el diagnóstico suele ser tardío (Segura, 2007). Considerando que las diferencias de desarrollo cerebral del SA no se restringen exclusivamente a cuestiones citoarquitectónicas sino también a los períodos de desarrollo, es necesario realizar estudios centrados en adultos con el objeto de caracterizar el procesamiento del lenguaje por el cerebro SA maduro.

El presente estudio tiene por objeto indagar, desde una perspectiva neuropsicolingüística, en el desempeño de adultos con SA en tres tareas lingüísticas que, en mayor o menor grado, implican un componente pragmático: comprensión de actos de habla indirectos, comprensión de metáforas y procesamiento de coerción aspectual. Mientras los dos primeros han sido ampliamente estudiados, aunque mayoritariamente en niños, el tercero es un fenómeno relativamente nuevo en la bibliografía, que no se ha estudiado, hasta donde sabemos, en sujetos con SA. La selección de estos tres fenómenos obedece a que, mientras el primero es canóni-

que entre ellos se presentan. En términos estadísticos, se asume que el sujeto 'neurotípico' es aquel que presenta las características más comunes, mientras el 'neurodivergente' es aquel que se aleja de la norma (Armstrong, 2012). En este contexto, cuando se habla de que las personas con SA presentan una alta capacidad de adaptación, nos referimos a que, aun teniendo un desarrollo cerebral 'neurodivergente', pueden llegar a tener una conducta 'neurotípica', en lo relativo a conductas sociales y comunicativas en general. 
camente pragmático, el segundo se vincula también con el procesamiento léxico y el tercero, con el morfosintáctico. El trabajo se organiza del siguiente modo. En primer lugar, se sintetiza lo establecido en la bibliografía acerca del componente pragmático en el procesamiento de los tres fenómenos en estudio, con especial atención a las investigaciones de sujetos con SA. Luego, se describe el método utilizado para la recolección y análisis de los datos. Más tarde, se exponen los resultados; y, finalmente, se presenta una discusión general seguida de conclusiones. Los resultados se interpretan desde la teoría de la relevancia de Sperber y Wilson (1995), modelo enmarcado en una pragmática inferencial que propone que el oyente interpreta el enunciado del hablante con el objeto de reconocer su intención comunicativa. De acuerdo con el modelo, el hablante realiza un acto ostensivo - por ejemplo, profiere un enunciado en determinado contexto- y el oyente infiere el significado más relevante, es decir, el que presenta la mayor informatividad -esto es, los mayores efectos cognitivos positivos- con el menor costo de procesamiento.

\section{EL PROCESAMIENTO PRAGMÁTICO DE ACTOS DE HABLA INDIRECTOS, METÁFORAS Y ORACIONES COERCIONADAS}

Los actos de habla indirectos se caracterizan como enunciados que obligan al oyente a realizar una inferencia para determinar su fuerza ilocutiva (Searle, 1975; Belinchón, Rivière e Igoa, 1992); más específicamente, en ellos, a diferencia de lo que ocurre con los actos de habla directos, el tipo de estructura léxico-gramatical del enunciado y su contenido ilocutivo no se corresponden de manera exacta. Así, una misma fuerza ilocutiva, como pedir al oyente que diga qué hora es, puede expresarse de distintas formas, ya como un acto de habla directo, como en Por favor, dime la hora, cuya forma es imperativa, ya como un acto de habla indirecto, como en ¿Me podrías decir la hora?, cuya forma es de pregunta, o en Necesito saber la hora, cuya forma es declarativa. Siguiendo la propuesta seminal de Searle (1975), para que una persona interprete de manera correcta un acto de habla indirecto, es necesario que realice procedimientos inferenciales atendiendo a la información pragmática disponible con el objeto de derivar la fuerza ilocutiva real de la estructura formal que escucha o lee $e^{5}$ Aun cuando podría pensarse que la comprensión de actos de habla indirectos debiera ser de suyo más compleja que la de los directos, diversos estudios muestran que, en determinados contextos, la comprensión de los primeros puede ser tanto o más rápida que la de los segundos (Gibbs y

\footnotetext{
${ }^{5}$ No todos los autores concuerdan con la caracterización searleana de los actos de habla indirectos. Así, por ejemplo, Morgan (1978) ha propuesto que se trataría de usos convencionales. Para un contraste de las distintas aproximaciones al problema de los actos de habla indirectos, véanse Escandell Vidal (1996) y Sadock (2006).
} 
Colston, 2006), lo que sugiere que los actos de habla indirectos no implican necesariamente, al menos en sujetos neurotípicos, mayor costo cognitivo que los directos. Especial relevancia tienen, en particular en el estudio de las habilidades pragmáticas de los sujetos con SA, las peticiones indirectas, toda vez que, por razones de cortesía (Brown y Levinson, 1987), los sujetos neurotípicos las emplean frecuentemente en sus interacciones cotidianas (Sadock, 2006). La dificultad para comprender este tipo de actos indirectos puede afectar de modo significativo las interacciones entre sujetos con SA y neurotípicos en el mundo real.

Por su parte, un enunciado metafórico puede describirse como un uso no literal del lenguaje, caracterizado por la correspondencia entre aspectos pertenecientes a dos dominios conceptuales distintos (Lakoff, 1993) ${ }^{6}$. Como en el caso anterior, la comprensión de metáforas depende del manejo correcto de la información pragmática (Cacciari y Glucksberg, 1994; Gibbs y Colston, 2006). El sustrato pragmático que subyace a su entendimiento puede caracterizarse, en términos griceanos, a partir de una violación intencional de la máxima de calidad, que desencadena en el hablante un proceso inferencial con el objeto de encontrar el verdadero sentido de la expresión (Grice, 1991). Por el contrario, desde la teoría de la relevancia la comprensión de metáforas no supone habilidades o procesos interpretativos cualitativamente distintos de los empleados en la comprensión de expresiones literales (Sperber y Wilson, 1995). En la presente investigación se consideran dos tipos de expresiones metafóricas. Por un lado, aquellas en que se explicita una relación de identidad entre un concepto fuente y otro meta -expresiones del tipo ' $\mathrm{X}$ es $\mathrm{Y}$ '- $\mathrm{y}$, por otro, aquéllas consistentes en unidades léxicas pluriverbales que no presentan composicionalidad plena, esto es, expresiones idiomáticas (Osorio, 2014). Mientras en el primer caso se presentan expresiones metafóricas que se pueden considerar relativamente nuevas, en el segundo se trata de expresiones convencionales.

Por último, la coerción aspectual consiste, típicamente, en un fenómeno de reinterpretación contextual en que, a partir de la incompatibilidad entre el aspecto léxico o inherente del verbo y su contexto de aparición (Michaelis, 2004), se genera un cambio de tipo implícito que altera el aspecto léxico. En el presente trabajo se sigue la categorización de tipos de situación de Vendler (1967), que distingue entre estados, logros, actividades y realizaciones, de acuerdo con los parámetros dinamismo, duración y telicidad o límite. En este marco, el aspecto léxico tradicio-

\footnotetext{
${ }^{6}$ Existe, como se sabe, una muy extensa bibliografía sobre la metáfora. Tradicionalmente, se la ha concebido como un tropo, esto es, una de las figuras de significación caracterizadas por el cambio de sentido de las palabras (Marchese y Forradellas, 1994). Frecuentemente, se ha propuesto que las metáforas descansan en relaciones de semejanza o aun en comparaciones implícitas (McGlone, 1996). Lakoff distingue entre expresiones metafóricas y metáforas conceptuales. Mientras las primeras son objetos lingüísticos, las segundas estructuran el campo conceptual, entendido como un dominio experiencial. Desde esta perspectiva, un enunciado metafórico es expresión lingüística de una metáfora conceptual.
} 
nal se caracteriza composicionalmente a partir de rasgos para cuya determinación se consideran no solo el verbo sino también sus argumentos (Smith 1997). Mientras los estados son no dinámicos, los otros tipos son dinámicos y se distinguen por los restantes parámetros. Así, los logros son télicos y no durativos, o puntuales; las actividades, durativas y atélicas, y las realizaciones, durativas y télicas.

La bibliografía distingue entre coerción aspectual exocéntrica y endocéntrica (Michaelis, 2004). La primera responde al modelo de oraciones como Pedro corrió en veinte minutos, en que la frase preposicional impone una reinterpretación télica, esto es, limitada o con punto de término, sobre el verbo correr que, de suyo, es atélico (una actividad ${ }^{7}$ ), sin operar directamente sobre éste. La segunda sigue el modelo de oraciones como María estaba ganando la carrera, en que la perífrasis de gerundio, operando directamente sobre el verbo, fuerza una reinterpretación estativa, esto es, no dinámica y durativa, sobre ganar, un logro dinámico y puntual. La presente investigación se centra en la coerción exocéntrica y adhiere a la postura pragmatista, según la cual, aun cuando la reinterpretación aspectual es motivada por una incompatibilidad de corte semántico, la solución de esta incongruencia se da mediante un proceso inferencial en que, a partir de los datos que provee el contexto y el consecuente reconocimiento de la intención comunicativa, se modifica la propiedad incompatible del ítem léxico, acomodándolo a las restricciones de la construcción o esquema gramatical con que entraba en conflicto (Michaelis, 2004; Smith, 1997).

\section{MÉTODO}

\subsection{Muestra}

La muestra (G1) estuvo conformada por cinco sujetos con SA, hablantes nativos de español de Chile, tres hombres y dos mujeres, cuyas edades fluctuaron entre los 18 y los 38 años. Para la conformación del grupo control (en adelante, G2), se procuró neutralizar las variables sexo, edad, nivel de escolaridad, ocupación, especialidad (área académica o laboral), diagnósticos asociados, medicamentos y lateralidad (zurdo vs. diestro). Adicionalmente, mediante evaluación fonoaudiológica, se neutralizaron las variables desarrollo cognitivo y desarrollo lingüístico general. Con el objeto de evaluar el primero, los sujetos fueron sometidos al Test de Matrices Progresivas de Raven (Raven y Court, 1993). Para el segundo, se emplearon el Test de Vocabulario y el Ítem Ideativo Complejo del Test de Boston (Goodglass y Kaplan, 2005) y el módulo de sintaxis de la Batería de Lenguaje (2011).

Para una discusión crítica más amplia del modelo y sus desarrollos posteriores, véase Soto 
Objetiva y Criterial (BLOC) (Puyuelo, Wiig, Renom y Solanas, 1997) ${ }^{8}$.

En este punto se hace indispensable reparar en la cantidad de individuos que conforman la muestra y su grado de representatividad de la población total portadora de SA. Ante todo, se debe considerar que, en los estudios neuropsicolingüísticos, las muestras se validan no solo por la cantidad de sujetos contemplados en ellas, sino, fundamentalmente, por un compromiso del desarrollo cerebral común a todos los sujetos que forman parte de la población estudiada. En este sentido, aunque la cantidad de individuos que conforman la muestra a observar en esta investigación no ascienda a un número que posea adecuación estadística (es decir, 30 o más), la evidencia neurológica es suficiente para que ésta sea representativa de la población total.

\subsection{Instrumentos}

Los sujetos seleccionados fueron sometidos a tres pruebas para evaluar su comprensión de actos de habla indirectos, metáforas y oraciones coercionadas, respectivamente. Tanto la de actos de habla como la de interpretación de metáforas se extrajeron de la estandarización argentina del Protocolo para la Evaluación de la Comunicación de Montreal (MEC) (Ferreres et al., 2007). Ambas pruebas constaron de dos tipos de estímulos: la de actos de habla presentó situaciones donde se proferían actos de habla directos y situaciones donde se proferían actos de habla indirectos, mientras la de interpretación de metáforas exigía interpretar metáforas nuevas y expresiones idiomáticas. Para la coerción aspectual se utilizó una prueba diseñada por Reyes (2012), que constó de tres cuestionarios de seis preguntas de alternativas cada uno.

\subsubsection{Prueba de actos de habla y de metáforas}

La prueba de actos de habla del MEC consta de situaciones donde se profieren tanto actos de habla directos (p. ej. Mariana mira con orgullo su nuevo Peugeot estacionado en la calle y le dice a su marido: "Me encanta el color que elegimos") como actos de habla indirectos con fuerza ilocutiva de petición (p. ej. Luisa mira su Peugeot sucio estacionado en la calle y le dice a su marido: “'No te parece que está demasiado sucio?”). El sujeto en todas las situaciones debe responder la pregunta: “¿Qué piensas que quiere decir la persona?". Cada situación tiene dos alternativas de respuesta: una literal y otra inferencial, siendo, en los actos de habla directos, correcta la literal, y en los indirectos, la inferencial.

\footnotetext{
${ }^{8}$ Agradecemos a los fonoaudiólogos Marcia Toloza y Rodrigo Tobar por la aplicación de las pruebas de evaluación y la elaboración de informes fonoaudiológicos de los sujetos.
} 
La prueba de enunciados metafóricos del MEC consta, por una parte, de metáforas nuevas, correspondientes a expresiones aún no convencionalizadas en español, con la forma "A es B", donde A y B son sustantivos (p. ej., Mi madre es una joya $)^{9}$. Además, incluye expresiones idiomáticas con la forma sujeto-verbocomplemento (p. ej. Aquel empleado es la mano derecha del jefe). Cada uno de los enunciados metafóricos es seguido por tres opciones de respuesta que plantean distintas interpretaciones: una literal, una figurada correcta y una interpretación metafórica errónea.

En ambas pruebas se solicitó a los sujetos que, en cada caso, otorgaran una respuesta antes de conocer las alternativas, asignándose 2 puntos a las respuestas claras y adecuadas; 1 a aquellas en que hay elementos de la respuesta presentes pero existen imprecisiones, agregados u omisiones, y 0 a los casos de respuesta errónea o ausencia de respuesta. Las dos pruebas fueron aplicadas a cada sujeto de G1 y de G2 por separado, durante aproximadamente 30 minutos, en una habitación aislada de ruidos exteriores. Como no se consideraron las variables tiempo, memoria de trabajo, ni atención, se explicaron las instrucciones y leyeron las situaciones a los entrevistados cuantas veces fuera necesario. El puntaje de cada sujeto se asignó según las instrucciones del test. Aun cuando el MEC considera la comparación del puntaje obtenido por cada sujeto con la norma establecida en la estandarización, tomando en cuenta la edad y el nivel de escolarización, esto no se realizó ya que la norma está pensada para sujetos de 30 años o más, edades superiores a la de la mayor parte de los sujetos que participaron en este estudio. La comparación del rendimiento de los sujetos de G1 se realizó con el de G2.

\subsubsection{Prueba de coerción aspectual}

La prueba de coerción aspectual consta de tres cuestionarios de seis preguntas cada uno, en los que se solicita a los sujetos leer un enunciado y, considerando que ninguna alternativa califica como correcta o incorrecta, escoger la opción que más se acerque al significado de la oración. Todos los cuestionarios presentan las mismas oraciones-estímulo (p. ej., "corrió en cinco minutos"), difiriendo, exclusivamente, en el contexto discursivo en que aparecen. La totalidad de los enunciados está construida con verbos de actividad como correr ([+dinámico], [+durativo], [-télico]), sujeto tácito (tercera persona singular) y un modificador temporal que exige la propiedad aspectual [+télico] (a saber, 'en 15 minutos'). Asimismo, todos están conjugados en pretérito simple del indicativo, adoptando una perspectiva perfectiva. El hecho de que se complemente el núcleo predicativo con un circunstancial de tiempo que exige el valor [+télico] desencadena un cambio de tipo aspectual

${ }^{9}$ La expresión metáfora nueva se emplea en el MEC en el sentido de no convencional; en este sentido, puede tratarse de metáforas usuales, como la del ejemplo. 
que puede devenir en una interpretación de realización ([+dinámico], [+durativo], [+télico]) o de logro ([+dinámico], [-durativo], [+télico]).

El primer cuestionario (en adelante, CI) consiste en la presentación de los enunciados sin contexto discursivo. Para cada estímulo se presentaron cuatro alternativas, dos de ellas correspondientes a interpretaciones derivadas de procesos de coerción que cambian el tipo aspectual del núcleo predicativo a un tipo télico, en un caso realización y en el otro logro. Mientras en la interpretación de realización debe sustituirse el rasgo [- télico] por [+ télico], en la de logro es necesario, además, sustituir [+ durativo] por [- durativo]. Las otras alternativas consisten, una, en una interpretación en que se mantiene el tipo aspectual de actividad del verbo, sin considerar la restricción télica del complemento -esto es, no se coerciona-, y la otra, en un enunciado que da cuenta de que los individuos no son capaces de recuperar el significado de la construcción sintáctica; en otras palabras, no comprenden. En el segundo cuestionario (en adelante, CII), los estímulos se presentan en un contexto discursivo que favorece una interpretación de realización; mientras que en el tercer cuestionario (CIII), se dan en un contexto discursivo que favorece interpretación de logro. En estos cuestionarios se presentan cinco alternativas, pues se agrega un distractor. La Tabla I presenta ejemplos de los estímulos en cada cuestionario:

Tabla I. Ejemplos de estímulos en los distintos cuestionarios del test de coerción aspectual.

\section{Cuestionario I: sin contexto discursivo \\ - Corrió en 15 minutos.}

\section{Cuestionario II: contexto sesgado a realización}

- "Juan tiene una rutina muy sana. Todas las mañanas se levanta muy temprano $\mathrm{y}$, antes de irse a trabajar, corre de su casa al parque, ida y vuelta. Ayer corrió en 15 minutos, por lo que llegó muy emocionado a su oficina”.

Cuestionario III: contexto sesgado a logro

- “Ana se compró una máquina trotadora. Hoy en la mañana, muy emocionada, la programó para correr por 30 minutos. Luego, buscó sus zapatillas, se puso un buzo, llenó una botella de agua e hizo algunas elongaciones para prevenir desgarros. Finalmente, corrió en 15 minutos". 
Para efectos de la presente investigación, se consideró la frecuencia de selección de cada alternativa en G2 para asignarle un puntaje entre 0 y 3, donde la opción con mayor frecuencia de selección recibe 3 y la opción con menor frecuencia 0 . No se asignó puntaje a la alternativa de no comprensión, que se analizó solo de forma cualitativa. La aplicación del instrumento se efectuó vía correo electrónico, con un mínimo de cinco días de diferencia entre la aplicación de cada uno de los cuestionarios. No se controló el tiempo de ejecución de la tarea en ninguno de los grupos.

\subsection{Análisis}

Tras la recolección de los resultados, se realizó un análisis cuantitativo que constó de dos fases: a) comparación de la media (en adelante, $\mathrm{M}$ ) y la desviación estándar (desde ahora, DE) del rendimiento de cada grupo en cada prueba, y b) aplicación de la prueba estadística no paramétrica de Mann-Whitney, mediante el uso del programa de análisis estadístico Prism ${ }^{\circledR}$. Posteriormente, se hizo un análisis cualitativo, en que se prestó especial atención a casos relevantes con el objeto de proponer una interpretación coherente de los resultados.

\section{RESULTADOS}

\subsection{Actos de habla}

El análisis estadístico de las pruebas de actos de habla muestra que el rendimiento de G1 y de G2 en la prueba de actos de habla directos fue muy similar, cercano al puntaje máximo posible $(\mathrm{M}=1,70$ y $\mathrm{DE}=0,37$ vs. $\mathrm{M}=1,78$ y $\mathrm{DE}=0,46$, respectivamente), con un valor-P de 0,318. Esto contrasta con lo que ocurre en la prueba de actos de habla indirectos, en que G1 obtuvo resultados más dispersos y significativamente inferiores $(\mathrm{M}=0,94$ y $\mathrm{DE}=0,40)$ a los de $\mathrm{G} 2(\mathrm{M}=1,92$ y $\mathrm{DE}=0,17)$, con un valor-P de 0,0001. Al comparar las dos pruebas de G1, se observa que los resultados de actos de habla indirectos son significativamente inferiores a los de actos de habla directos (valor- $\mathrm{P}=0,0014$ ). Contrastando las de $\mathrm{G} 2$, en cambio, se observa que, aunque el rendimiento es casi el mismo, en los actos de habla indirectos los resultados se encuentran menos dispersos (valor- $\mathrm{P}=0,5845$ ). Los siguientes gráficos ilustran los resultados: 
RLA. Revista de Lingüística Teórica y Aplicada, 53 (1), I Sem. 2015

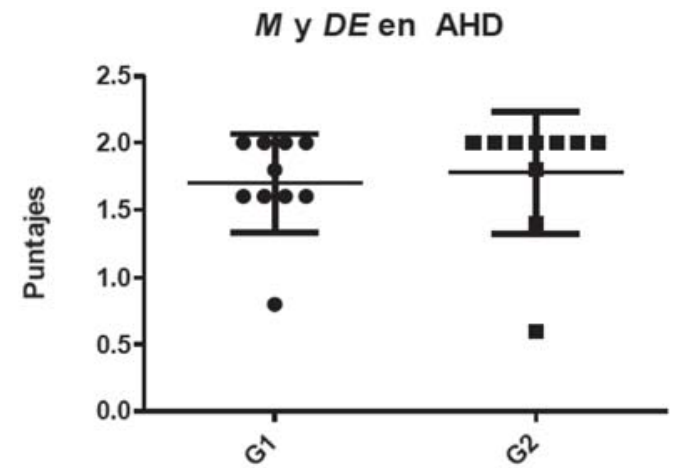

Gráfico 1. Media y desviación estándar de G1 y G2 en la interpretación de actos de habla directos (AHD).

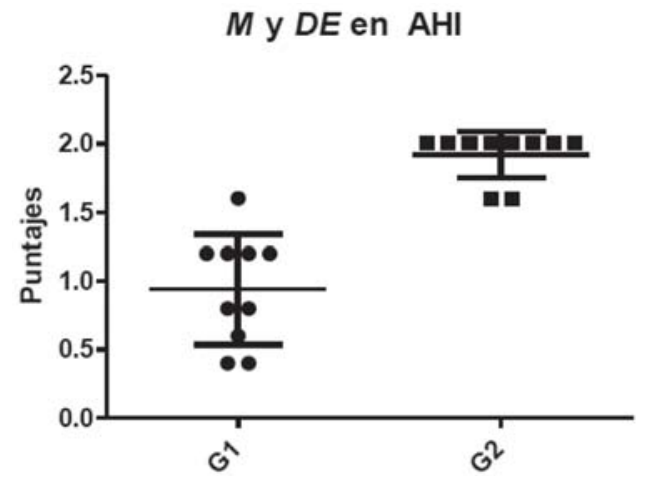

Gráfico 2. Media y desviación estándar de G1 y G2 en la interpretación de actos de habla indirectos (AHI).

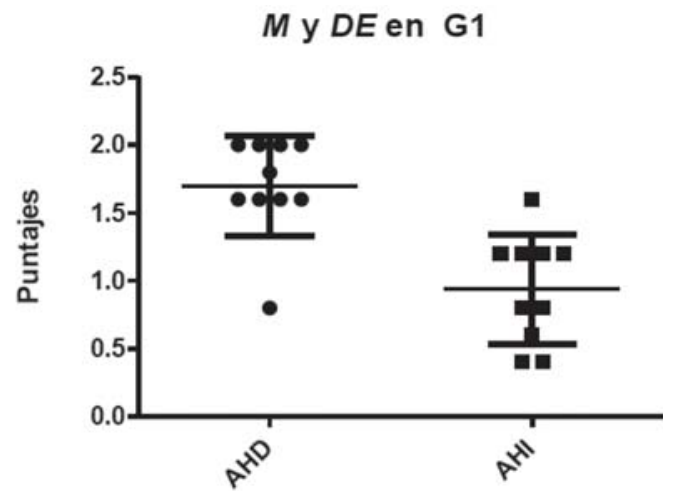

Gráfico 3. Media y desviación estándar de G1 en la interpretación de actos de habla directos (AHD) e indirectos (AHI). 
El componente pragmático en adultos con síndrome de Asperger: actos de... / C. Murray, A. Tobar, F. Villablanca, G. Soto

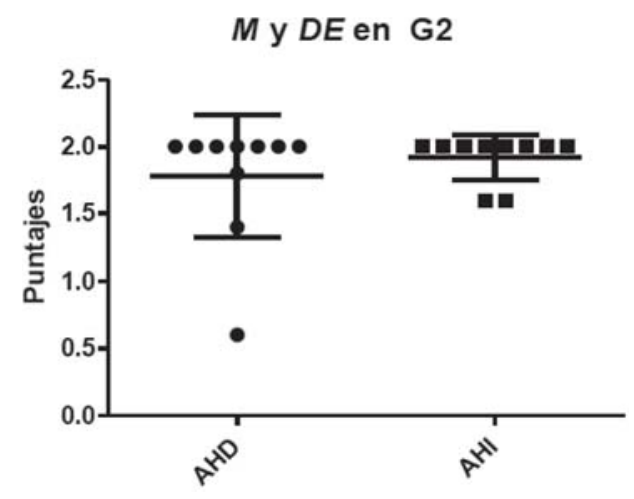

Gráfico 4. Media y desviación estándar de G2 en la interpretación de actos de habla directos (AHD) e indirectos (AHI).

Cualitativamente, encontramos en G1 dos tipos de error en el procesamiento de actos de habla indirectos. El primero de ellos, y más generalizado, corresponde a casos en que los sujetos realizaron indiscriminadamente interpretaciones literales de los enunciados. Por ejemplo, en el ítem "Luisa mira su Peugeot sucio estacionado en la calle y le dice a su marido: ¿No te parece que está demasiado sucio? ¿Qué piensas que quiere decir Luisa?", aunque la interpretación más adecuada del acto de habla sería "Luisa quiere que su marido lave el auto", en G1 se dan respuestas como "que el auto está sucio", "que está sucio el auto" o "que está sucio".

El segundo tipo de error corresponde a casos en que los sujetos logran, vía una inferencia, identificar una necesidad en el hablante, pero no dan cuenta del efecto que éste busca producir en el oyente para solucionarla. El siguiente ejemplo ilustra el error: "Juan está en su habitación escuchando música a un volumen muy alto. Su padre le dice Juan, me duele la cabeza. ¿Qué piensas que quiere decir el padre de Juan?". La respuesta esperada es "quiere que Juan baje o apague la música”; en G1, sin embargo, figura la respuesta "que no quiere escuchar música". Otro ejemplo: "Martín se sienta en el living a mirar televisión. Le dice a su mujer que está en la cocina: Mis anteojos están sobre la mesa. ¿Qué piensas que quiere decir Martín?”. Mientras la respuesta esperada es "quiere que su mujer le pase los anteojos", en G1 se responde "que no puede ver bien televisión".

\subsection{Metáforas}

En cuanto a la prueba de Interpretación de metáforas en general, existe una diferencia estadísticamente significativa entre G1 y $G 2(\mathrm{M}=1,14$ y $\mathrm{DE}=0,35$ vs. 
$\mathrm{M}=1,91$ y $\mathrm{DE}=0,17$, respectivamente), con un valor-P de 0,0001 , como se observa en el siguiente gráfico:

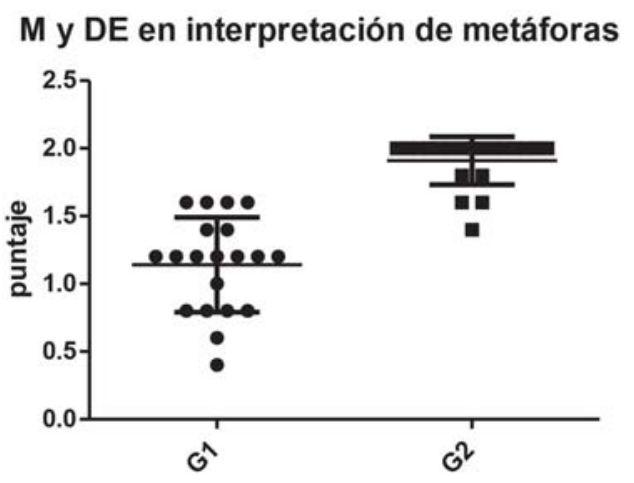

Gráfico 5. Media y desviación estándar de G1 y G2 en la interpretación de metáforas.

La diferencia entre los grupos y al interior de G1 es más acusada en el caso de la interpretación de expresiones idiomáticas que en la de metáforas nuevas. En efecto, en las primeras $\mathrm{G} 1$ presenta una $\mathrm{M}$ de 0,98 con una $\mathrm{DE}$ de 0,32 , mientras que $\mathrm{G} 2$ tiene una $\mathrm{M}$ de 1,92 con una $\mathrm{DE}$ de 0,17 , con un valor-P de 0,0001 . Por su parte, en la interpretación de las segundas, G1 tiene una $\mathrm{M}$ de 1,30 con una DE de 0,32, mientras que G2 tiene una M de 1,90 con una DE de 0,19, con un valor-P de 0,0005. Ambas situaciones se ilustran en los gráficos siguientes. Junto a lo anterior, se observa una diferencia importante en el rendimiento de G1 en ambas tareas (valor-P de 0,0429), en contraste con G2 en que la diferencia es escasa.

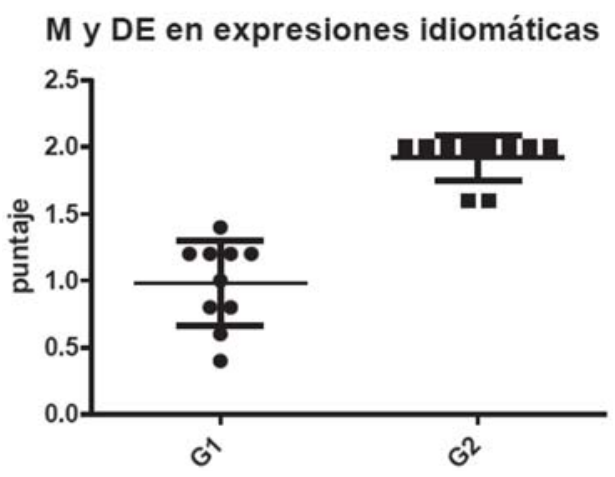

Gráfico 6. Media y desviación estándar de G1 y G2 en la interpretación de expresiones idiomáticas. 
El componente pragmático en adultos con síndrome de Asperger: actos de... / C. Murray, A. Tobar, F. VillablanCa, G. Soto

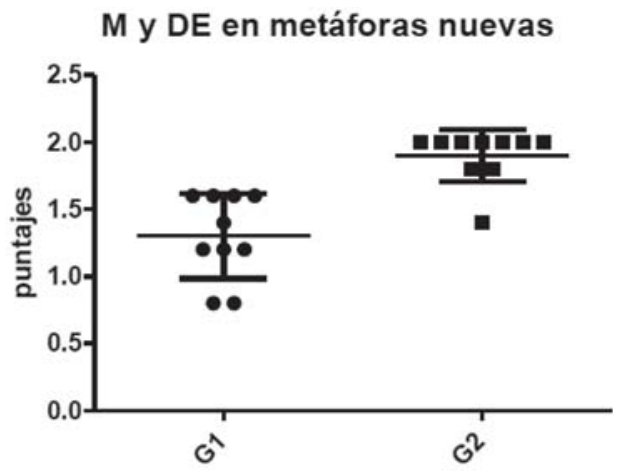

Gráfico 7. Media y desviación estándar de G1 y G2 en la interpretación de metáforas nuevas.

Es posible que la mayor complejidad que presenta la comprensión de expresiones idiomáticas para G1 obedezca a que estos estímulos, a diferencia de los de las metáforas nuevas, no tienen claves sintácticas que ayuden a la interpretación. En efecto, los enunciados con metáforas nuevas poseen una forma atributiva (con presencia de un verbo copulativo que relaciona dos entidades), que podría funcionar como una pista sintáctica, indicándole a los sujetos que se encuentran frente a un enunciado metafórico y orientándolos, consecuentemente, a una interpretación de este tipo. Por el contrario, los enunciados con expresiones idiomáticas no poseen una estructura fija que pueda operar como clave sintáctica. Por otro lado, es también posible que los dominios conceptuales sean más fáciles de vincular en los enunciados con metáforas nuevas que en los con expresiones idiomáticas. Como ya se ha indicado, en el MEC se consideran nuevas las metáforas no convencionalizadas, independientemente de su uso. Así, entre estos estímulos se encuentran expresiones bastante comunes como "mi hijo es un santo", "tu amiga es una víbora” o "ese alumno es un burro", que podrían ser más fáciles de comprender que cláusulas con expresiones idiomáticas como "mi marido me salió con un cuento chino" o "la maestra le habla a la pared".

\subsection{Coerción aspectual}

El rendimiento de G1 en la prueba de coerción aspectual es, en términos generales, significativamente inferior al de $\mathrm{G} 2(\mathrm{M}=8,7$ y $\mathrm{DE}=4,3$ vs. $\mathrm{M}=12,1$ y $\mathrm{DE}=2,9$, respectivamente), con un valor-P de 0,03 . Esta diferencia descansa en la importante diferencia que se observa en la ejecución de ambos grupos en CII, donde el contexto sesga a realización. En efecto, en CI la diferencia entre ambos grupos es 
pequeña: mientras en G1 se da una M de 11,4 y una DE de 4,3, en G2 hay una $\mathrm{M}$ de 12 y una DE de 3,6, con un valor-P de 0,8, esto es, no significativo. Aunque mayor, tampoco es significativa la diferencia en la ejecución de CIII, donde G1 presenta una $M$ de 9 y una DE de 5,1, mientras que G2 tiene una M de 11,8 y una DE de 5,3, con un valor-P de 0,58 . En contraste, en CII las diferencias de rendimiento entre G1 y G2 son significativas, con un valor-P de 0,01 ( $\mathrm{M=6,8}$ y $\mathrm{DE}=2,5$ vs. $\mathrm{M}=12,6$ y $\mathrm{DE}=1,2$, respectivamente). Los siguientes gráficos ilustran los resultados expuestos.

M y DE en prueba de coerción

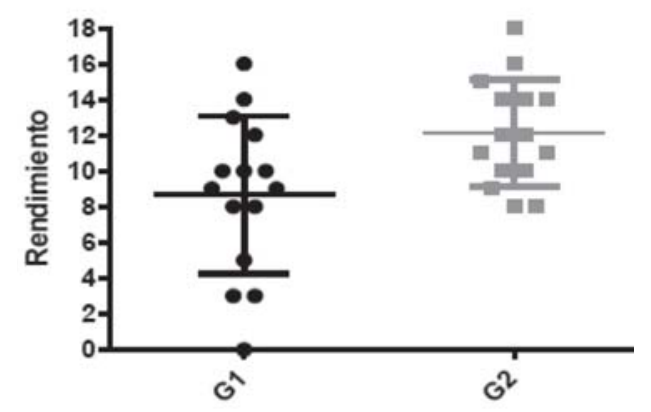

Gráfico 8. Media y desviación estándar de G1 y G2 en la comprensión de coerción aspectual (prueba completa).

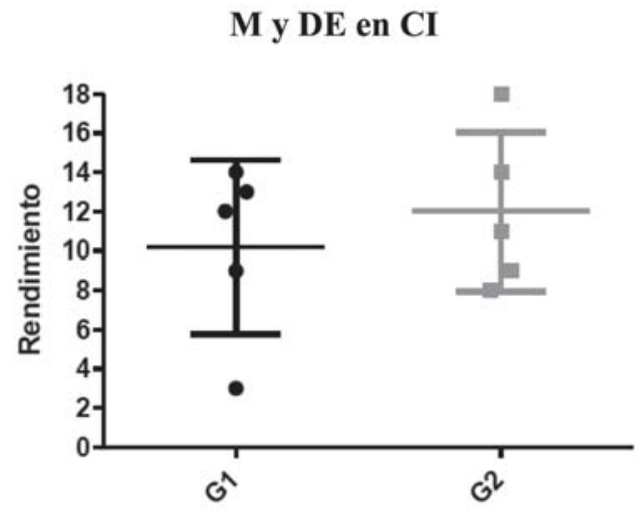

Gráfico 9. Media y desviación estándar de G1 y G2 en la comprensión de coerción aspectual (CI). 
El componente pragmático en adultos con síndrome de Asperger: actos de... / C. Murray, A. Tobar, F. Villablanca, G. Soto

\section{M y DE en CII}

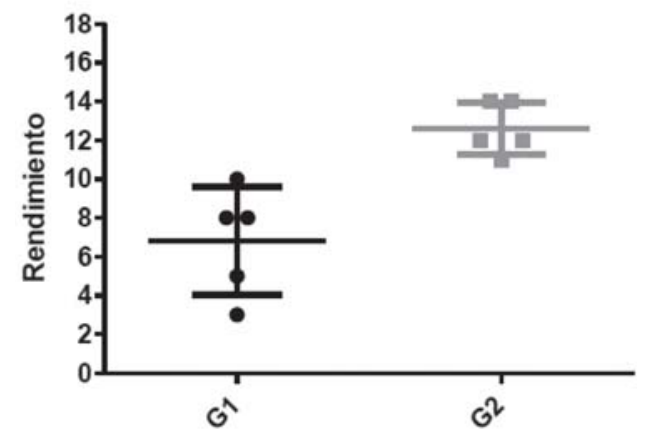

Gráfico 10. Media y desviación estándar de G1 y G2 en la comprensión de coerción aspectual (CII).

\section{M y DE en CIII}

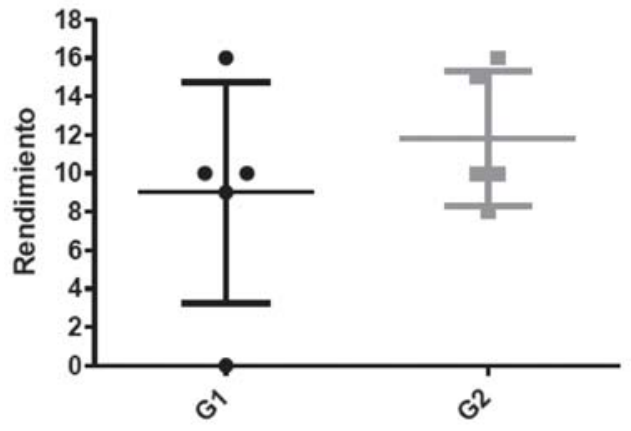

Gráfico 11. Media y desviación estándar de G1 y G2 en la comprensión de coerción aspectual (CIII).

Más allá del análisis de significatividad estadística, si se analiza el porcentaje de selección de cada alternativa en cada cuestionario por cada grupo, las diferencias parecen relevantes. Como se puede observar en la Tabla II, que expone los porcentajes de selección de la alternativa con la mayor asignación de puntaje (3) y de la alternativa "me parece incoherente" $\left({ }^{*}\right)$, tanto a nivel general como en cada uno de los tres cuestionarios, G2 presenta un mayor porcentaje de selección de la alternativa con mayor puntaje. Asimismo, mientras el porcentaje de selección de la alternativa "me parece incoherente" disminuye en G2 cuando los estímulos son presentados en contexto, en el caso de G1 no se presenta diferencia significativa 
entre ambas condiciones. Los datos parecieran sugerir que el rendimiento de G1 manifiesta diferencias cualitativamente importantes con respecto al de G2, específicamente en lo relativo al manejo y procesamiento de información contextual para la comprensión del significado de los estímulos.

Tabla II. Frecuencias relativas de selección de la alternativa 3 y de la alternativa "me parece incoherente" en los tres cuestionarios, comparando el rendimiento de G1 y G2.

\begin{tabular}{|c|c|c|c|c|}
\hline \multirow{2}{*}{ Cuestionario } & \multicolumn{2}{|c|}{ G1 } & \multicolumn{2}{c|}{ G2 } \\
\cline { 2 - 5 } & 3 & $*$ & 3 & $*$ \\
\hline CI & $40 \%$ & $23,3 \%$ & $53,3 \%$ & $23,3 \%$ \\
\hline CII & $20 \%$ & $26,6 \%$ & $53,3 \%$ & $0 \%$ \\
\hline CIII & $36,6 \%$ & $26,6 \%$ & $43,3 \%$ & $10 \%$ \\
\hline
\end{tabular}

\section{DISCUSIÓN Y CONCLUSIONES}

Considerados globalmente, los resultados de las tres pruebas sugieren, aunque en distinta forma y con distinta fuerza, un problema en el procesamiento o integración de la información contextual realizada por los adultos con SA. Esto es compatible con las teorías que plantean que la comprensión, en estos casos, requiere, en mayor o menor medida, de un adecuado procesamiento de información pragmática, tarea especialmente difícil para estos sujetos. En efecto, la comprensión de actos de habla indirectos supone que el sujeto, considerando la información contextual que entrega el estímulo, deseche la interpretación directa. El contraste entre la conducta de G1 en esta tarea y en la comprensión de actos de habla directos, cuya interpretación depende de aspectos formales, indica que, probablemente, los sujetos con SA, a diferencia de los neurotípicos, no utilizan adecuadamente la información contextual para resolver el problema. En lo que respecta a la comprensión de metáforas, si bien en esta prueba los estímulos no se presentan contextualizados, los resultados son compatibles con la tesis de que es necesario recurrir a información pragmática para recrear mentalmente una situación comunicativa en la que éstos cobren sentido. Finalmente, el que G1 haya tenido un rendimiento diferente a G2 en tareas de coerción -en particular cuando éstas implicaban procesamiento de información contextual que sesgaba a favor de un tipo de coerción- sugiere, también, un déficit en el procesamiento de la información pragmática. 
Desde el punto de vista lingüístico, el déficit en el procesamiento de información contextual podría caracterizarse como un mal funcionamiento del principio de relevancia (Sperber y Wilson, 1995). De acuerdo con éste, durante la comprensión discursiva, el oyente debe concentrar su atención en los elementos que resultan más pertinentes para la comprensión, esto es, aquellos que exigen bajo esfuerzo de procesamiento, al mismo tiempo que entregan información crucial para identificar el significado global. Es posible pensar que, en el caso de G1, hay una dificultad para identificar los elementos más relevantes del contexto en tiempo real, lo que se traduciría en una falta de consideración de elementos contextuales o en una selección inadecuada de los mismos.

Desde el punto de vista neurocognitivo, los resultados son compatibles con al menos tres de las teorías sobre el SA propuestas por la bibliografía especializada. Por una parte, el comportamiento de G1 es concordante con la teoría de la coherencia central (Frith, 2003; Happé, 1999), que plantea que los sujetos con autismo y SA, a diferencia de los neurotípicos, centran su atención en los constituyentes particulares y no en el contenido global de una situación. Esto implicaría un procesamiento exhaustivamente detallado en que no se reconoce el contenido o significado global (Rajendran y Mitchell, 2007); en otras palabras, un déficit en la comprensión de la coherencia global. Tanto las dificultades para integrar información contextual en la comprensión de actos de habla indirectos y oraciones coercionadas, como el posible empleo de claves sintácticas -esto es, estructuralesen la comprensión de metáforas sugieren dificultades como las expuestas por esta teoría.

Los resultados también pueden interpretarse desde la teoría hipersistematizante, según la cual los sujetos con SA, al carecer de un desarrollo normal de la empatía y de la teoría de la mente, tienden a comprender la realidad como sistema de reglas, es decir, tienen una disposición a observar las partes constituyentes de la información con el fin de identificar y aplicar las reglas internas de un sistema (Baron-Cohen, 2002; Baron-Cohen y Belmonte, 2005). Esta conducta hipersistematizante podría explicar no solo que el contexto no funcione como facilitador en el test de coerción, sino también el privilegio del sentido literal en las pruebas de actos de habla y el posible empleo de claves sintácticas en el test de metáforas que ya hemos comentado. Más aún, podría especularse que la disposición a hipersistematizar se vincula con el déficit en la coherencia central. Por un lado, puede pensarse que la tendencia a observar los constituyentes en busca de reglas atenta en contra de la identificación del significado global; por otro, que un déficit en la coherencia central favorece el desarrollo de una estrategia hipersistematizante.

Como se ha dicho, también se ha caracterizado el SA por un déficit en el desarrollo de la teoría de la mente, esto es, en la habilidad para atribuir estados mentales a otros y reconocer estados mentales propios (Baron-Cohen et al., 1985; Happé, 1994). La interpretación desde esta teoría de los resultados en las pruebas 
de comprensión de actos de habla indirectos y metáforas es bastante clara. En la primera, la identificación adecuada de la petición indirecta en cada situación supone la habilidad de atribuir estados mentales a los interlocutores involucrados en el proceso comunicativo, una tarea en que el grupo G1 tiene un comportamiento deficiente. Es sugerente que, incluso en los casos en que enfrentados a actos de habla indirectos, los sujetos con SA infieren estados mentales que van más allá de la información explícita, éstos no sean los que asignaría un sujeto neurotípico. En las pruebas de metáforas, por su parte, en tanto parece necesario representarse mentalmente una situación comunicativa en que la emisión del enunciado cobre sentido; también se requeriría de la habilidad de teorizar acerca de los estados mentales de los agentes que participan en la comunicación. Finalmente, si bien los resultados de la prueba de coerción no apoyan directamente esta teoría, es posible proponer una relación entre ambos si se acepta, como han propuesto Pylkkänen, Martin, McElree y Smart, (2009), que el proceso de reinterpretación contextual que supone la coerción se realiza en la zona frontal ventromedial, zona que también participa de la teoría de la mente y que se relaciona con el procesamiento de información pragmática (Baars y Gage, 2007). En la medida en que el déficit en el desarrollo de la teoría de la mente podría favorecer el desarrollo de una estrategia hipersistematizante en los sujetos con $\mathrm{SA}$, es posible que las tres explicaciones neurocognitivas no sean excluyentes sino que se refuercen entre sí.

En síntesis, los resultados muestran que el comportamiento de los adultos con SA es distinto del de los neurotípicos. Más específicamente, sugieren que, a diferencia de lo que ocurre con éstos, en los sujetos con SA la información contextual no influye positivamente en el proceso de comprensión. Asimismo, se puede inferir que las tres habilidades lingüísticas estudiadas poseen un componente pragmático y que la diferencia entre ambos grupos podría obedecer a un déficit en la operación del principio de relevancia en los adultos con SA, hipótesis cuya verificación, con todo, requiere de estudios ulteriores. En lo que respecta a las teorías de funcionamiento neurocognitivo del SA, los resultados son concordantes tanto con la teoría hipersistematizante como con la de mal funcionamiento del sistema de coherencia central y, de modo más indirecto, con la de déficit en la teoría de la mente.

\section{REFERENCIAS}

Armstrong, Thomas. 2012. El poder de la neurodiversidad. Barcelona: Paidós. Attwood, Tony. 2007. Guía del sindrome de Asperger. Barcelona: Paidós.

Baars, Bernard J. y Nicole M. Gage. 2007. Cognition, brain and consciousness: an introduction to cognitive neuroscience. Amsterdam: Academic Press.

Baron-Cohen, Simon. 2002. "The extreme male brain theory of autism", en 
El componente pragmático en adultos con síndrome de Asperger: actos de... / C. Murray, A. Tobar, F. Villablanca, G. Soto

Trends in Cognitive Science 6, pp. 248-254.

Baron-Cohen, Simon y Matthew Belmonte. 2005. "Autism: a window onto the Development of social and the analytic brain", en Annual Review of Neuroscience 28, pp. 109-126.

Baron-Cohen, Simon, Alan Leslie y Uta Frith. 1985. "Does the autistic child have a theory of mind?”, en Cognition 21, pp. 37-46.

Belinchón, Mercedes, Ángel Rivière, y José Igoa. 1992. Psicología del lenguaje. Investigación y teoría. Madrid: Trotta.

Brown, Penelope y Stephen Levinson. 1987. Politeness. Some universals in language use. Cambridge: Cambridge University Press.

Cacciari, Cristina y Sam Glucksberg. 1994. "Understanding figurative language". En Morton Ann Gernsbacher (ed.) Handbook of psycholinguistics. San Diego, CA: Academic Press.

Escandell Vidal, M. Victoria. 1996. Introducción a la pragmática. Barcelona: Ariel.

Ferreres, Aldo, Valeria Abusamra, Macarena Martínez-Cuitiño, Bernardette Ska, Hélène Côté e Yves Joanette. 2007. Protocolo para la Evaluación de la Comunicación de Montréal (MEC). Buenos Aires: Neuropsi.

Frith, Uta. 2003. Autism: Explaining the enigma. 2a ed. Oxford: Blackwell.

Ghaziuddin, Mohammad y Leonore Gerstein. 1996. "Pedantic speaking style differentiates Asperger syndrome from high-functioning autism”, en Journal of Autism and Developmental Disorders 26, 6, pp. 585-595.

Gibbs, Raymond W. y Herbert L. Colston. 2006. "Figurative language". En Matthew J. Traxler y Mornton A. Gernsbacher (eds.) Handbook of psycholinguistics. 2a ed. Oxford: Academic Press.

Goodglass, Harold y Edith Kaplan. 2005. Evaluación de la afasia y de trastornos relacionados. (Test de Boston). Madrid: Médica Panamericana.

Grice, Paul. 1991. "Lógica y conversación”. En Luis Valdés (ed.) La buisqueda del significado. Lecturas en filosofia del lenguaje. Madrid: Tecnos.

Hadjikhani, Neda. 2007. Progress in Autism Research. EE.UU.: Nova Science Publishers.

Happé, Francesca. 1994. "An advanced test of theory of mind -understanding of story characters thoughts and feelings by able autistic, mentally- handicapped, and normal- children and adults", en Journal of Autism and Developmental Disorders 24, 2, pp. 129-154.

Happé, Francesca. 1999. "Autism: cognitive deficits or cognitive style?”, en Trends in Cognitive Science 3, 6, pp. 216-222.

Kaland, Nils, Annette Møller-Nielsen, Kirsten Callesen, Erik Lykke Mortensen, Dorte Gottlieb, and Lars Smith. 2002. "A new advanced test of Theory Of Mind. Evidence from children and adolescents with Asperger Syndrome", en Journal of Child Psychology and Psychiatry 43, 4, pp. 517-528.

Lakoff, George. 1993. "The contemporary theory of metaphor". En Andrew 
Ortony (ed.) Metaphor and thought. 2a ed. Cambridge: Cambridge University Press.

Le Sourn-Bissaoui, Sandrine, Stephanie Caillies, Fabien Gierski y Jacques Motte. 2009. "Inference processing in adolescents with Asperger syndrome: relationships with theory of mind abilities", en Research in Autism Spectrum Disorders 3, pp. 797-808.

Lewis, Fiona, Bruce Murdoch y Gail Woodyatt. 2007. "Linguistic abilities in children with autism spectrum disorders", en Research in Autism Spectrum Disorders 1, pp. 85-100.

Loukusa, Soile e Irma Moilanen. 2009. "Pragmatic inference abilities in individuals with Asperger syndrome or high-functioning autism. A review", en Research in Autism Spectrum Disorders 3, pp. 890-904.

Marchese, Angelo y Joaquín Forradellas. 1994. Diccionario de retórica, crítica y terminología literaria. Barcelona: Ariel.

McAlonan, Grainne, John Suckling, Naikei Wong, Vinci Cheung, Nina Lienenkaemper, Charlton Cheung y Siew Chua. 2008. "Distinct patterns of grey matter abnormalily in high-functioning autism and Asperger's syndrome", en Journal of Child Psychology and Psychiatry 49, 12, pp. 1287-1295.

McGlone, Matthew. 1996. "Conceptual metaphors and figurative language interpretation: food or thought?", en Journal of Memory and Language 35, 544-565.

Michaelis, Laura. 2004. "Type shifting in construction grammar: an integrated approach to aspectual coercion”, en Cognitive Linguistics 15(1), pp. 1-67.

Morgan, Jerry. 1978. "Two types of convention in indirect speech acts". En Peter Cole (ed.) Syntax and semantics. Vol 9: Pragmatics. Nueva York: Academic Press.

Murray, Constanza, Anita Tobar y Fanny Villablanca. 2012. Procesamiento pragmático en sujetos con síndrome de Asperger: actos de habla indirectos, metáforas y coerción aspectual. Informe final de Seminario para optar al grado de Licenciado en Lengua y Literatura Hispánica con mención en Lingüística. Santiago, Chile: Universidad de Chile.

Norbury, Courtenay. 2005. "Barking up the wrong tree? Lexical ambiguity resolution in children with language impairments and autistic spectrum disorders", en Journal of Experimental Child Psychology 90, 2, pp. 142-171.

O'Brien, Finian, Lisa Page, Ruth O'Gorman, Patrik Bolton, Ajay Sharma, Gillian Baird, Eileen Daly, Brian Hallahan, Ronán Conroy, Catherine Foy, Sarah Curran, Dene Robertson, Kieran Murphy y Declan Murphy. 2010. "Maturation of limbic regions in Asperger syndrome: a preliminary study using proton magnetic resonance spectroscopy and structural magnetic resonance inaging", en Psychiatry Research: Neuroimaging 184, pp. 77-85.

Osorio, Gabriela. 2014. Comprensión y producción de expresiones idiomáticas en sujetos con síndrome de Asperger. Tesis para optar al grado de Magíster en Lingüística Mención Lengua Española. Santiago, Chile: Universidad de Chile. 
Pellicano, Elizabeth. 2007. "Links between theory of mind and executive function in young children with autism: clues to developmental primacy", en Developmental Psychology 43, 4, pp. 947-990.

Pijnacker, Judith, Peter Hagoort, Jaan Buitelaar, Jan-Pieter Teunisse y Bart Geurts. 2008. "Pragmatic Inferences in High-Functioning Adults with Autism and Asperger Syndrome", en Journal of Autism and Developmental Disorders 39, pp. 607-618.

Puyuelo, Miguel, Elisabeth Wiig, Jordi Renom y Antoni Solanas. 1997. Batería de Lenguaje Objetiva y Criterial (BLOC). Barcelona: Masson.

Pylkkänen, Liina, Andrea Martin, Brian McElree y Andrew Smart. 2009. "The anterior midline field: Coercion or decision making?”, en Brain \& Language 208, pp. 184-190.

Rajendran, Gnanathusharan y Peter Mitchell. 2007. "Cognitive theories of autism”, en Developmental Review 27, pp. 224-260.

Raven, John y John Court. 1993. Test de Matrices Progresivas. Escala Coloreada. Buenos Aires: Paidós.

Reyes, Catalina. 2012. Coercion on the edge: A purely linguistic phenomenon or an integrated cognitive process. Tesis de Magíster en Estudios Cognitivos. Santiago, Chile: Universidad de Chile.

Rivière, Ángel. y José Castellanos. 2003. "Autismo y teoría de la mente”. En Mercedes Belinchón, Alberto Rosa, María Sotillo, Inés Marichalar y Ángel Rivière (eds.) Obras Escogidas. Vol. II. Lenguaje, simbolización y alteraciones del desarrollo. Madrid: Panamericana.

Sadock, Jerrold. 2006. “Speech acts”. En Laurence R. Horn y Gregory Ward (eds.) The handbook of pragmatics. Oxford: Blackwell.

Searle, John. 1975. "Indirect Speech Acts". En Peter Cole y Jerry L. Morgan (eds.) Syntax and semantics. Vol. 3: Speech acts. Nueva York: Academic Press.

Segura, Hugo. 2007. Discurso narrativo, paisaje de la conciencia y teoría de la mente en sujetos con síndrome de Asperger. Tesis de Magíster en Estudios Cognitivos. Santiago, Chile: Universidad de Chile.

Shriberg, Lawrence, Rhea Paul, Jane MacSweeny, Ami Klin, Donald Cohen y Fred Volkmar. 2001. "Speech and prosody characteristics of adolescents and adults with high-functioning autism and Asperger syndrome", en Journal of Speech, Language, and Hearing Research 44, pp. 1097-1115.

Smith, Carlota. 1997. The parameter of aspect. 2a ed. Dordrecht: Kluwer.

Soto, Guillermo. 2011. Los tiempos compuestos del indicativo en el español hablado en América. Tiempo, aspecto y uso. Tesis doctoral. Valladolid, España: Universidad de Valladolid.

Sperber, Dan y Deirdre Wilson. 1995. Relevance: communication and cognition. 2a ed. Oxford: Blackwell.

Thompson, Lynda, Michael Thompson y Andrea Reid. 2010. "Functional neu- 
RLA. Revista de Lingüística Teórica y Aplicada, 53 (1), I Sem. 2015

roanatomy and the rationale for using EEG biofeedback for clients with Asperger's syndrome", en Applied Psychophysiology and Biofeedback 35, pp. 39-61.

Valdez, Daniel. 2005. "Teoría de la mente, memoria autobiográfica y síndrome de Asperger. Fundamentos para la intervención clínica y educativa”,en Revista el Cisne 179, pp. 1-8.

Vendler, Zeno. 1967. "Verbs and times". En Zeno Vendler (ed.) Linguistics in philosophy. Nueva York: Cornell University Press. 